
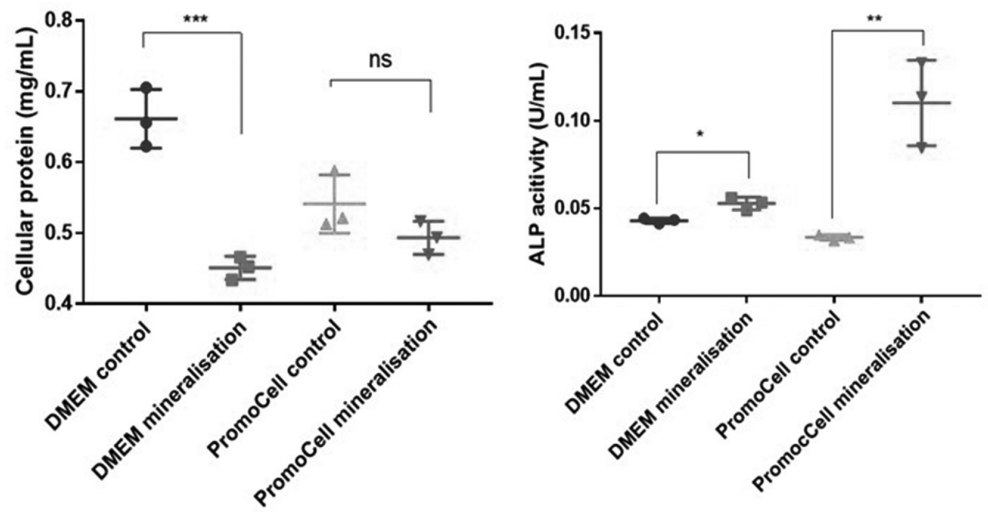

Abstract BS51 Figure 2 Quantification of cellular protein and ALP activity after 21 days of culture in DMEM mineralisation media or PromoCell mineralisation media and respective controls. ${ }^{*}$ indicates significantly different to control $\left({ }^{*} p<{ }^{* *} p<0.01,{ }^{* * *} p<0.001\right)$

aortic smooth muscle cells (HASMCs). Hypoxic conditions were examined as it was recently shown that hypoxic signalling plays a role in the progression of VC during CKD.

Methods HASMCs (P4-5; PromoCell) were grown in 6- or 12 -well cell culture plates until about $70 \%$ confluence in regular commercial smooth muscle cell growth media (PromoCell). The cells were then: 1) Cultured for 21 days in DMEM mineralisation media (10nM dexamethasone and $10 \mathrm{mM}$ sodium $\beta$-glycerophosphate) or control media, 2) Cultured for 21 days in commercial mineralisation media or control media (PromoCell), 3) Cultured for 4, 6, and 8 days in high phosphate media (PromoCell; $2.5 \mathrm{mM}$ PO4) or control media in hypoxic conditions ( $1 \%$ O2), 4) Cultured for 6 days in commercial mineralisation media (PromoCell) or control media in hypoxic conditions, or 5) Cultured for 8 and 12 days in DMEM mineralisation media or control media in hypoxic conditions. Alizarin red staining was used to detect calcification at the end of the experiments, cell morphology was examined, and alkaline phosphatase (ALP) activity and total protein content were measured.

Results Alizarin red staining showed that DMEM mineralisation media cultured cells were completely calcified after 21 days and very faint staining was visually seen in DMEM control media cultured cells (protocol 1; figure 1). PromoCell mineralisation media cultured cells were moderately calcified (protocol 2) and control cells in PromoCell regular media maintained the characteristic morphology of HASMCs, while mineralised cells were characteristic of transdifferentiated, calcifying HASMCs (protocol 2, figure 1). DMEM mineralisation media (protocol 1) significantly decreased cell protein after 21 days compared to DMEM control media cultured cells, while this did not occur in PromoCell media treated cells (protocol 2; figure 2). PromoCell mineralisation media and DMEM mineralisation media both significantly increased ALP activity in cell lysates compared to respective controls (figure 2). No calcification was detected in cells subjected to the hypoxic conditions (protocols 3-5).

Conclusion Culturing cells in PromoCell mineralisation media for 21 days in normoxic conditions was evaluated to be the most suitable method for inducing calcification in HASMCs.
Hypoxia failed to induce calcification in HASMCs in any of the tested media conditions.

Conflict of interest None

\section{BS52 THE EFFECTS OF IGFBP2 OVER-EXPRESSION ON VASCULAR FUNCTION AND SMOOTH MUSCLE}

${ }^{1}$ Thomas Slater*, ${ }^{2}$ Alexander Bruns, ${ }^{2}$ Stephen Wheatcroft, ${ }^{1}$ Mark Kearney. ${ }^{1}$ Leeds Institute of Cardiovascular and Metabolic Medicine, The University of Leeds; ${ }^{2}$ University of Leeds

\subsection{6/heartjnl-2019-BCS.213}

Introduction Insulin-like growth factor binding protein 2 (IGFBP2) plays an important role in cell proliferation and migration in numerous cell types. However, little is known about the effects of IGFBP2 on vascular function.

We have previously demonstrated IGFBP1 over-expression upregulates endothelial nitric oxide (NO) generation through PI3/AKT activation, leading to reduced atherosclerosis and blood pressure. IGFBP2 has been shown to stimulate angiogenesis through activation of the PI3/AKT pathway. Therefore over-expression of IGFBP2 may also have positive effects on endothelial NO generation.

To test this, we investigated vascular function using novel transgenic mouse models of tamoxifen-inducible whole body overexpression of IGFBP2, and tamoxifen-inducible selective endothelial overexpression of IGFBP2. Subsequently we examined the effects of IGFBP2 stimulation on human vascular smooth muscle (VSMC) proliferation.

Methods Cre-lox recombination was used to create hIGFBP2 tamoxifen-inducible mice. This involved the creation of transgenic mice with cDNA encoding hIGFBP2 but with a floxed stop codon preventing transcription, on a C57BL/6 background. These were bred with VE-Cad-ERT-Cre mice. When these mice are injected with tamoxifen at 8 weeks, the Cre recombinase is activated, cleaving the stop codon and allowing transcription of hIGFBP2 in the vascular endothelium alone. These mice were termed VEhIHom. The same floxed hIGFBP2 mice were also bred with CMV-CreERT2 mice. Following tamoxifen injection, 